\title{
EXPERIENCIA DE REFLEXIÓN SOBRE EL PORTAFOLIO DE DESEMPEÑO PEDAGÓGICO (PDP) EN LA EVALUACIÓN DEL DOCENTE EN CHILE
}

\section{REFLECTION EXPERIENCE IN THE PORTAFOLIO OF PEDAGOGICAL PERFORMANCE IN TEACHERS' EVALUATION IN CHILE \\ Paola Vidal Sepúlveda Alejandra Nocetti de la Barra}

Magister en Ciencias de la Educación, Universidad Católica de la Santísima Concepción, Chile. p.vidalsepulveda@uandresbello.edu

\section{RESUMEN}

La política pública apunta a un proceso de evaluación con carácter formativo, pero no existe suficiente investigación al respecto y, por ello, el objetivo del estudio fue describir la experiencia de reflexión en el 
marco de la elaboración del portafolio de desarrollo pedagógico (PDP), en profesores de establecimientos públicos en la comuna de Santiago, Chile. El estudio fue interpretativo - particularmente un estudio de casos-, en que participaron docentes seleccionados mediante muestreo intencional. Se desarrollaron entrevistas semiestructuradas, sometidas a un análisis de contenido. Los resultados muestran condiciones obstaculizadoras de la reflexión de carácter personal e institucional. Se identificaron menos condiciones facilitadoras de la reflexión y se concluye que existen más condiciones que limitan la reflexión y también podría advertirse que no se cumple la función formativa de esta evaluación docente.

Palabras clave: evaluación docente, práctica reflexiva, docentes en servicio.

\section{ABSTRACT}

Public policy points to an evaluation process with a formative nature, but there is not enough research in this regard and therefore, the objective of the study was to describe the experience of reflection in the framework of the development of the portfolio of pedagogical performance (PDP) in teachers of public establishments in the commune of Santiago, Chile. The study was interpretive, particularly a case study, in which teachers selected by intentional sampling participated. Semi-structured interviews were developed, which were subjected to a content analysis. The results show impeding conditions for personal and institutional reflection. Less facilitating conditions for reflection were identified and it is concluded that there are more conditions that limit reflection and also, it could be noted that the formative function of this teaching evaluation is not fulfilled.

Keywords: teacher evaluation, reflexive practice, teachers in service.

En Chile existe evidencia, a través de diagnósticos nacionales (Agencia de Calidad de la Educación, 2015; Centro de Estudios Mineduc, 2018; Eyzaguirre y Le Foulon, 2016), que todavía no se alcanzan los estándares de aprendizaje comprometidos en la política pública a 
nivel nacional. En dicho contexto, uno de los factores estudiados con interés son las características del profesorado, clave para el aprendizaje del estudiantado (Darling-Hammond, 2012; Eyzaguirre S., 2019; Santelices, Galleguillos, González y Taut, 2015; Schmelkes, 2014).

En esta línea se iniciaron investigaciones acerca de la influencia que ejerce el profesorado como actor relevante en el proceso de aprendizaje (Barber y Mourshed, 2008; Danielson, 2011; Gordon, Kane y Steiger, 2006; Nye, Konstantopoulos y Hedges, 2004); los estudios establecen una relación entre los resultados del SIMCE (Sistema de Medición de la Calidad Educativa) y las características del profesorado (Bravo, et al., 2008; León, Manzi y Paredes, 2008; Toledo, 2010). Al respecto, surge una creciente tendencia a valorar la calidad del profesorado (Calatayud, 2014).

Algunos autores (Santelices, Galleguillos, González y Taut, 2015), explican que la calidad docente se enfoca en tres lineamientos: 1) Características observables del profesorado y su impacto en los aprendizajes. 2) Prácticas pedagógicas sustentadas en estándares de desempeño, medidos con criterios observables. 3) Estimaciones de valor agregado, basadas en el rendimiento de los estudiantes en pruebas estandarizadas.

La Evaluación de Desempeño Docente (EDD) chilena, integra estas tres dimensiones como medida única de calidad. Asimismo, el sistema evaluativo se articula con la carrera docente para promover la profesionalización del profesorado y la calidad de la educación (Ministerio de Educación, 2019).

Es un modelo de evaluación formativo, coincidente con Rosales (1999), quien señala que este tipo de evaluación tiene por objetivo el perfeccionamiento de funciones esenciales en el ejercicio de la profesión docente, y con Valdés (2000), autor que concibe a la evaluación como oportunidad de perfeccionamiento o avance en la actividad profesional.

Está regulada por los parámetros del Marco de la Buena Enseñanza, el cual establece criterios observables de un buen desempeño profesional docente; dichos estándares constituyen una influencia particular 
en el profesorado que evaluará su desempeño ya que, de acuerdo con especialistas, la evaluación orienta la actividad educativa y «determina el comportamiento de los sujetos, no solo por los resultados que pueda ofrecer, sino porque esta preestablece qué es lo deseable, qué es lo valioso, qué es lo que debe ser» (Bretel y Crespo, 2002, p. 25).

Valdés (2000) sitúa en el centro al profesorado para caracterizarlo y propiciar su desarrollo. Según esto, es acertado que el Ministerio de Educación (MINEDUC) incorpore el sistema de EDD como pilar de desarrollo profesional. Sin embargo, es necesario investigar si el instrumento PDP favorece un proceso de mejoramiento de la práctica docente y, en ese sentido, es relevante estudiar la reflexión en contexto de la EDD. Resultados presentados por el MINEDUC muestran que una de las ponderaciones más bajas en la evaluación docente se presenta en la dimensión reflexión crítica sobre la práctica (Ministerio de Educación, 2013; Pérez-Cotapos y Taut, 2016). Este antecedente, indica que debería desarrollarse investigación que explore la experiencia del profesorado, en particular con el PDP, en el cual se valora la reflexión pedagógica, a fin de disponer de información sobre el proceso de evaluación en sí mismo. Así, surge la interrogante de investigación para describir la experiencia de reflexión en el marco de la elaboración del PDP del profesorado en establecimientos públicos en la comuna Santiago, Chile.

\section{ANTECEDENTES TEÓRICO CONCEPTUALES}

\section{Contexto y génesis del sistema de evaluación}

La reforma educativa iniciada en la década de los noventa, en contexto de una reciente democracia, define una modificación de eje curricular y además un refuerzo a la profesionalización docente (Lemaitre, 1999 y Núñez, 2003, en Donoso, 2005) lo que implicó aumento de remuneraciones, pasantías en el exterior y cambios en la formación inicial, entre otras reformas (García-Huidobro y Cox, 1999). Pese a lo anterior, los informes y las pruebas internacionales revelan que no hay avances. Además, el perfeccionamiento del profesorado no dio los frutos esperados (Rubilar, 2004). 
Como respuesta se implementaron políticas educativas tendientes a modificar la capacitación del profesorado y asegurar la calidad de los resultados de los estudiantes, aplicando un sistema de calificaciones al profesorado (Ministerio de Educación, 2017). Sin embargo, y en contexto de negociaciones, en 1998, el magisterio acordó la suspensión del proceso evaluativo proveniente del Estatuto Docente (Assaél y Pavez, 2008; Assaél y Inzunza, 2008) y, a cambio, el gobierno se comprometía a elaborar, en conjunto con el profesorado, un sistema de EDD de acuerdo con una visión compartida (Assaél y Inzunza, 2008).

\section{La evaluación de desempeño docente}

La evaluación del desempeño docente debería permitir al profesorado conocer sus necesidades, que se convertirán en requerimientos de profesionalización o perfeccionamiento (Bonifaz, 2011; Centro de Perfeccionamiento, Experimentación e Investigaciones Pedagógicas, 2008; UNESCO, 2007). La preparación del instrumento PDP se inicia con la comprensión del estándar bajo el que será evaluado, es decir el MBE, el cual podría considerarse como un tipo de rúbrica o matriz.

En el ámbito teórico se reconocen dos tipos de rúbricas: holísticas y analíticas. Según Gatica y Uribarren (2013), la rúbrica holística realiza una valoración integrada del desempeño, pero no especifica los componentes del proceso, demanda menor tiempo para la calificación, pero su retroalimentación es menor. La rúbrica analítica descompone los desempeños en criterios y niveles de logro, que se definen con gran detalle, de modo que la retroalimentación recibida por los evaluados es muy minuciosa.

El MBE podría considerarse como una rúbrica holística en que se especifican las dimensiones a evaluar pero que, sin embargo, los indicadores de corrección se detallan a los pares correctores del PDP y no al profesorado (Sun, Correa, Zapata, y Carrasco, 2011). Dichos criterios se diseñan en rúbricas analíticas que crean los organismos externos encargados de corregir pero que, según expertos en evaluación, deberían ser explicitados a los evaluados como una forma de mayor comprensión y transparencia de lo esperado (Murillo, 2007; OCDE, 2014). 


\section{Evaluación de desempeño docente en contextos internacionales}

UNESCO (2007), afirma que existe una creciente necesidad en América Latina, como en Europa, de contar con criterios mensurables que objetiven, en conductas observables, el buen desempeño profesional del profesorado para promover una carrera horizontal.

En América Latina, los resultados de la evaluación del desempeño se emplean para el avance en la carrera profesional docente, es el caso de Argentina, Bolivia, Colombia, Costa Rica, México, entre otros; y en algunos países europeos como: Alemania, Eslovaquia, España, Estonia y Francia.

También es utilizado para el aumento de remuneraciones, lo que ocurre en Chile, Cuba y Honduras; y en cuatro países de Europa: Chipre, Eslovenia, Letonia y República Checa.

\section{MODELO DE EVALUACIÓN DE DESEMPEÑO CHILENO}

Especialistas (UNESCO, 2007) utilizan el propósito de la EDD como criterio de clasificación de los modelos de evaluación. De acuerdo con esto, el sistema de evaluación chileno se inserta en dos modelos: como base para un incremento salarial y como incentivo al avance profesional. El modelo para la promoción en el escalafón docente se relaciona con la reciente formulación de la carrera docente y los tramos de desarrollo profesional.

\section{Disposiciones de la Evaluación de Desempeño Docente}

El sistema de evaluación se rige por la Ley número 19.961 del año 2004, modificando y complementando el artículo 70 del Estatuto Docente; es formativo, con una periodicidad de cada cuatro años. Existen cuatro niveles de logro, a saber: destacado, competente, básico 
e insatisfactorio (Centro de Perfeccionamiento, Experimentación e Investigaciones Pedagógicas, 2008). Los instrumentos son:

\section{Tabla 1. Los instrumentos de evaluación del desempeño}

\begin{tabular}{|c|c|}
\hline $\begin{array}{l}\text { INSTRUMENTO } \\
\text { Y PONDERACIÓN }\end{array}$ & DETALLE \\
\hline $\begin{array}{l}\text { Autoevaluación } \\
10 \%\end{array}$ & $\begin{array}{l}\text { Pauta para reflexionar sobre su práctica pedagógica y } \\
\text { valorar su desempeño. }\end{array}$ \\
\hline $\begin{array}{l}\text { Entrevista } \\
\text { Evaluador } \\
\text { Par } 20 \%\end{array}$ & $\begin{array}{c}\text { Es una entrevista estructurada de aproximadamente una } \\
\text { hora con un evaluador par (docente de aula del mismo } \\
\text { nivel y modalidad del evaluado). }\end{array}$ \\
\hline $\begin{array}{l}\text { Informe } \\
\text { Referencia de } \\
\text { Terceros } \\
10 \%\end{array}$ & $\begin{array}{l}\text { Pauta estructurada que debe ser completada por el } \\
\text { Director y el Jefe de la Unidad Técnico Profesional } \\
\text { del establecimiento y contiene preguntas referidas al } \\
\text { quehacer del docente evaluado. }\end{array}$ \\
\hline $\begin{array}{l}\text { Portafolio de } \\
\text { desempeño } \\
\text { Pedagógico } \\
\text { —digital- } \\
60 \%\end{array}$ & $\begin{array}{l}\text { Recoge de forma online, a través de productos } \\
\text { estandarizados, evidencia verificable respecto de las } \\
\text { mejores prácticas de desempeño del docente evaluado. } \\
\text { Contempla la presentación de productos escritos, en los } \\
\text { cuales el docente debe reportar distintos aspectos de su } \\
\text { quehacer profesional y un registro audiovisual en vivo } \\
\text { consistente en la grabación en video de una clase de } \\
\text { cuarenta minutos de duración. }\end{array}$ \\
\hline
\end{tabular}

Fuente: Elaboración propia con base en bibliografía MINEDUC, 2019.

La corrección de las evidencias del PDP la realizan profesores correctores del mismo nivel y subsector de aprendizaje del docente evaluado. El portafolio, como instrumento de evaluación, concita el apoyo de expertos (Alfageme, 2007; Cisneros-Cohernour, 2008; Banta, 2003; Martin-Kniep, 2001; Seldin y Annis, 1990). 


\section{LA PRÁCTICA REFLEXIVA EN LA EDD}

La práctica reflexiva es entendida como el examen de los supuestos que subyacen a la acción docente en situaciones específicas de la enseñanza, reconociendo sus implicaciones éticas y políticas (Perrenoud, 2004). La práctica reflexiva se asocia a la reflexión sobre la acción, comprendida como el proceso mental que toma la propia acción como objeto de cuestionamiento y la reflexión en la acción, proceso que ocurre durante la acción profesional (Schön, 1992; Perrenoud, 2004).

Existe una vinculación entre los niveles de reflexión y el desarrollo profesional (Van Manen, 1977). Se plantea que existe una jerarquía que obedece a la racionalidad de la acción profesional que predomina en cada nivel. La reflexión técnica se distingue por el examen de la propia actuación, en términos de la efectividad de la aplicación del conocimiento educativo, para alcanzar unos fines externos. La reflexión práctica se refiere al interés por esclarecer los supuestos que subyacen al trabajo docente, juzgando las consecuencias éticas que conlleva la propia acción. Y finalmente, la reflexión crítica examina el trabajo del profesorado de acuerdo con criterios comprometidos con la justicia social (Domingo, 2008; Domingo et al., 2014).

El proceso formativo de evaluación contempla la reflexión profesional como un componente relevante, lo que lleva a reconocer que debería promover reflexión de carácter práctico o crítico, según la propuesta de Van Manen (1977). No obstante lo anterior, se advierte en otras investigaciones que el profesorado no demuestra altos niveles de reflexión en el proceso de evaluación pedagógica (Erazo, 2009), sino que se limita a un examen de fortalezas y debilidades, reforzando una reflexión de carácter técnico, no vinculada con la construcción de conocimiento en la acción ni con el cambio de práctica docente (Nocetti y Medina, 2019).

Lo anterior contradice a Guzmán et al. (2012), quien considera la práctica reflexiva como modelo de formación y, por lo tanto, debiera ser parte del proceso de evaluación del desempeño docente. Además, este autor subraya que se requiere de acompañamiento estimulador, porque el profesorado no sabe cómo llevar a cabo este proceso o también 
la confunde con términos afines, tal como sucede con los docentes en formación (Nocetti y Medina, 2019).

Por otra parte, como plantean Preiss et al. (2014), tener acceso a la observación de la propia actuación constituye una oportunidad para el aprendizaje reflexivo. Tal como señalan Nocetti y Medina (2018), la observación de la actuación del otro - mediada por un video- estimula la reflexión en dos direcciones; es decir, reflexiona quien expone su clase, pero también quienes participan del análisis. Otros autores establecen que los conocimientos que emergen de un análisis reflexivo entre pares, favorecen la construcción de conocimientos contextualizados pertinentes y significativos para los propios actores (Avalos, 2011; Korthagen, 2010). Estos autores, además, agregan que el conocimiento que emerge de la práctica analizada, la mayoría de las veces significa una mejora de la actuación profesional y creación de nuevo conocimiento. En esa perspectiva, la elaboración del portafolio - que incluye la filmación de una clase-debería incentivar el examen de la actuación docente entre pares profesionales.

\section{METODOLOGÍA}

El estudio se adscribió al paradigma interpretativo (Denzin y Lincoln, 2013), explorando significados que subyacen a la acción profesional (Flick, 2015), especialmente en la elaboración del portafolio de evaluación docente. Se analizaron casos particulares a partir de los cuales se derivaron conclusiones de carácter general (Martínez, 1996). Cabe destacar que la selección de los casos obedeció a su potencial de transferencia, dado que permite proyectar los resultados a otros contextos profesionales similares.

Como contexto, se indica que la investigación se desarrolló con profesores de la región metropolitana (capital de Chile), evaluados recientemente en la EDD, y del sistema público.

Conforme a la naturaleza cualitativa del muestreo, no se guió el número de informantes, no obedeció a criterios estadísticos, sino a saturación teórica (Gibbs, 2012), es decir, las conversaciones finalizaron al 
no emerger nuevos significados que permitieran un mayor desarrollo conceptual de las categorías configuradas en el marco de este estudio.

El caso es relevante porque existe escasa información respecto de la experiencia de reflexión en el marco de la EDD; solo se cuenta con evidencia que la reflexión demostrada por los docentes es de nivel bajo. Se utilizó como estrategia de recolección de datos la entrevista en profundidad (Kvale, 2011) y la entrevista focalizada (Canales, 2006) con profesores que, voluntariamente y con consentimiento informado, accedieron a participar de una serie de conversaciones sucesivas hasta lograr la saturación teórica de los datos.

El dato textual emergió de las entrevistas semiestructuradas, que fueron transcritas de acuerdo con criterios de Kvale (2011). Posteriormente, se realizó un análisis de contenido (Gibbs, 2012), que consideró varias etapas: 1) reducción de los datos en que se desarrolló la codificación abierta, levantándose categorías entendidas como unidades de significado de mayor nivel de abstracción; 2) transformación de datos mediante la elaboración de matrices y redes, dando origen a un conjunto de subcategoría; 3) obtención de resultados y conclusiones, a partir de la comprensión de la experiencia basada en la configuración de las unidades de significados, construidas en etapas anteriores (Rodríguez, Gil, y García, 1996).

La credibilidad, transferibilidad y auditabilidad se emplearon como criterios de calidad en esta investigación (Vasilachis, 2006).

Para cumplir con la credibilidad, se llevaron a cabo extensas entrevistas - transcritas textualmente - para evidenciar lo que los sujetos dicen y piensan respecto de la propia vivencia.

El criterio de transferibilidad se propició a través de información suficiente para emitir juicios de semejanzas y diferencias con otros contextos.

Finalmente, también se adoptó el criterio de auditabilidad. En este caso, el desarrollo del estudio estuvo guiado por una académica, especialista en el campo de la investigación en práctica reflexiva. 


\section{RESULTADOS}

Al explorar en la experiencia de elaboración del portafolio docente, se identificaron condiciones que facilitan y que obstaculizan el examen reflexivo de la propia actuación profesional.

\section{Categoría: condiciones facilitadoras de la reflexión}

Representa las condiciones que, de acuerdo con el profesorado, instan a desarrollar un examen de la propia acción de enseñanza. En general, puede afirmarse que se trata de factores indirectos y no propios de la elaboración del portafolio docente y que, por lo mismo, podrían generarse independiente del proceso EDD.

Uno de los factores es trabajar en un contexto vulnerable, ya que allí se enfrenta habitualmente baja efectividad del trabajo docente. Se suma a lo anterior, la presión por mejorar los resultados académicos sin considerar que, a veces, no se cuenta con las condiciones para lograr esa meta académica. Entonces, el apremio por responder a dichas exigencias institucionales y ministeriales genera cuestionamiento en el profesorado, lo que se acentúa en época de evaluación, pero no puede imputarse al proceso de evaluación en sí mismo, tal como se plantea:

- Entrevistador PV: ¿Qué aspectos del PEE le hicieron reflexionar?

- Yo no veo mucha reflexión en el portafolio. Quiero destacar que trabajar en este contexto municipal lo lleva a uno a reflexionar porque parece que todo aquí es más difícil y el ministerio no toma en cuenta esto, y sentimos la presión. Definitivamente, creo que es un factor determinante el contexto, porque en el fondo aquí hay que enfrentar diversos problemas y hay que demostrar que puedes sí o sí, hacer clases (Profesora Cc).

Por otra parte, el profesorado expresó que un detonante natural de la reflexión surge de circunstancias negativas e inesperadas en el diario quehacer dentro del aula y que, en este caso, pueden afectar la percepción del propio desempeño en el marco de EDD. De este modo, 
surge una «especie de trabajo colaborativo» que se activa a partir de la presión por demostrar cierta efectividad en la evaluación, tal como se ilustra a continuación:

- Entrevistador PV: Y a propósito de la elaboración del PEE. ¿Se estimula una práctica reflexiva? O sea, ¿entre ustedes? [...].

- En general no, pero como hay que hacer el portafolio, uno se preocupa más por la percepción de su propia actuación [...]. Entonces, sí lo hacemos en torno a cuestiones específicas: «Mira, me pasó algo con este niño». La profesora $\mathrm{T}$ a veces a mí me ha preguntado: «¿Qué podemos hacer con este alumno?». Y nos apoyamos para buscar soluciones y esto se vuelve más común conversarlo cuando estamos en evaluación docente (Profesora L).

El profesorado manifestó que el portafolio sirve como catarsis para expresar sentimientos negativos. En ese sentido, suscitan reflexión las condiciones en que se trabaja y eso, en ocasiones, desencadena frustración, especialmente en instituciones educativas vulnerables. Nuevamente se reitera una reflexión sobre aspectos que no se centran en la actuación profesional, sino en las condiciones laborales:

Yo lo que veo es que más que nada [que] el elaborar el portafolio sirve para desahogarnos: «[...] Nos fue mal», «[...] Las condiciones en que trabajamos», «[...] Estaba difícil la evaluación como cosas puntuales; pero que nos sentemos a reflexionar, no». Entonces, claro que se reflexiona, pero no sobre nuestra tarea, sino sobre las condiciones en que trabajamos (Profesora S).

En síntesis, no existen condiciones que el profesorado reconozca claramente como favorecedoras de la reflexión pedagógica y que estén vinculadas a la experiencia de la evaluación docente. Por el contrario, aparecen factores de carácter contextual que presionan a demostrar mayor efectividad profesional, evidenciándose un carácter técnico de la reflexión vinculada al proceso de evaluación. 


\section{Categoría: condiciones obstaculizadoras de la reflexión docente}

Representa factores que podrían obstaculizar la reflexión docente. Algunas condiciones se refieren a características del instrumento, la racionalidad técnica que caracteriza el proceso y la experiencia reflexiva del profesorado.

Uno de los obstaculizadores de la reflexión es la presión por demostrar efectividad didáctica o pedagógica, es decir, evidenciar cierto conocimiento teórico y habilidades docentes que se han estandarizado, y debido a este carácter técnico del proceso, queda poco espacio para la reflexión centrada en una perspectiva práctica:

No hay mucho espacio para la reflexión sobre lo vivido, uno tiene que demostrar ciertos conocimientos en Didáctica, manejar todo eso. A veces, eso te obliga a buscar en los libros [...] temas de la enseñanza o de la evaluación [...]. Uno tiende a escribirlo paso a paso, o bien, pedir ayuda externa para que quede bonito. Entonces, queda poco espacio para lo real y la reflexión en sí misma (Profesora L).

Otra condición que desincentiva la reflexión docente son las características del instrumento que guía el desarrollo del portafolio: el carácter estructurado y el tipo de preguntas que orienta la evaluación.

Se percibe que el instrumento es estructurado y existe escaso espacio para describir la propia experiencia docente real:

- Entrevistador P.V. ¿Qué aspectos del PEE le hicieron reflexionar?

- [...] Reflexionar no, porque es algo súper estructurado y eso: no tenemos la libertad para reflexionar, tampoco incentiva a pensar en la propia experiencia junto con los pares y ver que uno va aprendiendo; no se da esa instancia (Profesora T).

Además, se observan preguntas e instrucciones más bien orientadas a una autoevaluación. De este modo, las preguntas imponen un carácter instrumental a la evaluación y desincentivan una reflexión con carácter práctico, tal como se observa en el Portafolio 2019: 
Tarea 3. Reflexión: Análisis a partir de las características del estudiante. En esta tarea debe reflexionar sobre cómo incorporó las características de su grupo de estudiantes al planificar o realizar las clases de su Unidad Pedagógica (DocenteMás, 2019, p. 23).

Otro factor obstaculizador de la reflexión es que el profesorado reconoce que no tiene claro qué tipo de reflexión se espera de ellos. Esta falta de consenso del significado de la reflexión pedagógica provoca un sentimiento de minusvalía del proceso reflexivo:

Es que ahí caemos nuevamente, en otro problema con la reflexión, no sabemos qué quieren ellos en una reflexión. Porque para mí, puede ser una reflexión lo que yo expuse ahí, pero es mal evaluada. En definitiva: no se valora mí propia reflexión, eso es lo que yo veo (Profesora L).

Los profesores se esfuerzan por acertar con la forma en que se espera que ellos reflexionen y buscan ayuda de otros que obtuvieron buenos resultados en el ítem de reflexión docente. Entonces no se describe una reflexión vivida, sino que el profesorado está más pendiente de ajustarse a los instructivos ministeriales. De este modo, resultan dos consecuencias: no describen la verdadera experiencia reflexiva y tampoco reciben retroalimentación para mejorar esta competencia profesional:

No [es] una reflexión verdadera, no, porque te exigen reflexiones específicas, concretas, reflexiones que tengan palabras clave que a veces uno no maneja y entonces, uno busca ayuda con otros colegas a los que le fue bien en esta parte; lo malo es que a uno no lo logran retroalimentar verdaderamente, y entonces no se puede mejorar y, así, se va haciendo como un círculo vicioso (Profesora C).

Otro obstaculizador de la práctica reflexiva es que la mayoría del profesorado no dispone de tiempo y tampoco de espacios para hacerlo. Esta condición contextual, afecta evidentemente el desarrollo de la habilidad para reflexionar y eso no se considera en la evaluación del desempeño docente: 
Yo quisiera agregar, que lo otro que juega en contra, cuando se evalúa la reflexión en el portafolio, es que no se considera que en nuestra jornada de trabajo no hay tiempo y espacios para reflexionar con los colegas. Entonces, es difícil que sepamos cómo hacerlo y que vayamos desarrollando esa competencia. Esto no es considerado y se nos evalúa como si tuviéramos las condiciones ideales para hacerlo (Profesora C).

Finalmente, otro factor obstaculizador de la reflexión en la EDD, corresponde a la falta de experiencia reflexiva cotidiana, ya que esta tiende a ser esporádica y referida a la efectividad de la acción profesional. En ese sentido, la EDD podría constituirse en un espacio para desarrollar una práctica reflexiva:

Yo pienso que el portafolio debería ser algo continuo. No así [solo] una vez; tendría que ser un instrumento que acompañe, en que va registrando sus reflexiones y la forma en que va aprendiendo de este proceso de análisis de su práctica. Entonces, el propio instrumento no ayuda a hacer un seguimiento de los avances en materia de la habilidad para reflexionar y mejorar. En suma, trabaja en el portafolio la reflexión en base a una clase que se filma, lo hace, lo deja ahí y nada más... hasta el otro portafolio (Profesora D).

\section{HALLAZGOS Y DISCUSIÓN}

A partir de los hallazgos, puede afirmarse que el profesorado no reconoce condiciones facilitadoras de la reflexión, vinculadas a la elaboración del portafolio docente; por el contrario, sí reconocen obstaculizadores de la práctica reflexiva relacionados a la estructura y modalidad de la evaluación docente.

Los resultados permiten establecer que la forma en que se evalúa al profesorado promueve una reflexión técnica, alcanzando el nivel más bajo de la jerarquía que se propone para el desarrollo profesional (Van Manen, 1977). Esto se evidencia al reconocer el carácter estructurado del instrumento y el tipo de preguntas que guían el proceso evaluativo. 
Además podría deducirse, a partir de los hallazgos, un significado de reflexión limitado a un proceso de autoevaluación, coincidiendo con el enfoque formativo del profesorado (Nocetti y Medina, 2018).

Asimismo se observó que la modalidad de la evaluación lleva al profesorado a centrarse en demostrar conocimiento teórico y ajustarse a una práctica estandarizada, acentuando una racionalidad técnica. Esto coincide con lo señalado por Erazo (2009), estableciendo que, a nivel nacional, el profesorado ha perdido la habilidad para reflexionar críticamente sobre su propia actuación profesional.

Los profesores se esfuerzan por demostrar una alta coherencia entre su discurso pedagógico y los estándares establecidos por el MINEDUC. De este modo, podría afirmarse que la elaboración del PDP no genera suficiente espacio para reflexionar sobre lo vivido, ni tampoco suscita reflexión entre pares, perdiéndose oportunidades para enriquecer la propuesta ministerial a partir de la experiencia del profesorado.

Además, se observan condiciones contextuales que no se consideran en la EDD, como la falta de tiempo y de espacio para la reflexión profesional (Perrenoud, 2004; Tardif, 2004), que dificultan el desarrollo de esta competencia. Asimismo, reconocen dificultad para definir qué significa reflexionar o qué se espera cuando se les pide que reflexionen en el PDP. Los resultados indican que no reciben retroalimentación, lo que no permite mejorar la habilidad reflexiva, configurándose un círculo que mantiene al profesorado sin avanzar en esta competencia.

Los hallazgos denotan cierta pérdida del carácter profesional de la actuación docente, al reforzar miradas instrumentales de su evaluación. Esto no conduce al profesorado a desarrollar niveles más complejos de reflexión que signifiquen una mirada más crítica de la acción profesional y una transformación de lo educativo. Tampoco se está propiciando que este proceso les permita recuperar su condición de sujetos de conocimiento, tal como lo han planteado expertos con relatos de experiencias docentes (Contreras, 2016; Perrenoud, 2004). 
Los resultados del estudio develan cierta tensión, ya que la práctica educativa se adhiere a un enfoque constructivista promovido por la reforma educacional, lo que contradice el paradigma con que se evalúa al profesorado. Aparece así una incongruencia evaluativa a este nivel, esto es: evaluar el desempeño desde una perspectiva técnica y promover una valoración más auténtica con el estudiantado.

Finalmente, podrían proponerse mayores esfuerzos para que la EDD promueva una reflexión que incluya acompañamiento entre pares, mayores opciones de avanzar hacia una reflexión práctica y crítica (Van Manen, 1977) que favorezca una consciencia de aspectos de la práctica docente, que significan desigualdad e injusticia social.

Se reconoce como limitación del estudio, la imposibilidad de generalizar estos resultados al profesorado nacional, pero los casos analizados muestran una realidad que es preocupante respecto del rol reflexivo que debería cumplir el proceso de evaluación profesional y podría servir de base para desarrollar estudios futuros con un mayor alcance nacional.

\section{REFERENCIAS}

Agencia de Calidad de la Educación (2015). Reporte de Calidad Agencia de Calidad de la Educación. Santiago: MINEDUC.

Alfageme, M. (2007). «El portafolio reflexivo: metodología didáctica en el EEES». Revista Educatio Siglo XXI, 25, 209-226.

Assaél, J. e Inzunza, J. (2008). La actuación del Colegio de profesores. Buenos Aires: Laboratorio de Políticas Públicas.

Assaél, J., y Pavez, J. (2008). «La construcción e Implementación del Sistema de Evaluación del Desempeño Docente Chileno: principales tensiones y desafíos». RIEE. Revista Iberoamericana de Evaluación Educativa, 41-55. 
Avalos, B. (2011). «Teacher professional development in teaching and teacher education oven ten years». Teaching and Teacher Education, 27, 10-20.

Banta, T.W. (2003). Portfolio assessment: uses, cases, scoring and impact. Assessment update collection. San Francisco: Jossey-Bass.

Barber, M. y Mourshed, M. (2008). Cómo hicieron los sistemas educativos con mejor desempeño del mundo para alcanzar sus objetivos. McKinsey y Company.

Bonifaz, R. (2011). «El Origen de la Evaluación Docente en Chile». En J. Manzi, R. González y Y. Sun, La Evaluación Docente en Chile. Santiago: Centro de Medición MIDE UC, 13-34.

Bravo, D., Falck, D., González, R., Manzi, J. y Peirano, C. (2008). «La relación entre la evaluación docente y el rendimiento de los alumnos: Evidencia para el caso de Chile». Encuentro Anual de la Sociedad de Economía de Chile. Viña del Mar, Chile.

Bretel, L. y Crespo, E. (2005). La evaluación como medio para asegurar los aprendizajes. Recuperado de http:/ / breteleando.blogspot.com

Calatayud Salom, M.A. (2014). «Evaluación de la práctica docente y calidad educativa: una relación encadenada». Revista Iberoamericana de Educación, 65(1), 1-12.

Canales, M. (2006). Metodología de Investigación Social. Santiago.

Centro de Estudios Mineduc (2018). Estudio internacional TALIS Contextos de la enseñanza y el aprendizaje según las percepciones de profesores $y$ directores en Chile. Santiago.

Centro de Perfeccionamiento, Experimentación e Investigaciones Pedagógicas (2008). Marco para la Buena Enseñanza, séptima edición. Santiago: MINEDUC.

Cisneros-Cohernour, E. (2008). «El portafolio como instrumento de evaluación docente. Una experiencia del sureste de México». Revista Iberoamericana de Educación. ISSN:1989-0397. 
Contreras, J. (2016). «Relatos de experiencias, en busca de un saber pedagógico». Revista Brasileira de Pesquisa (Auto) Biográfica, Salvador, $1,14-30$

Danielson, C. (2011). «Competencias docentes: desarrollo, apoyo y evaluación». Programa de Promoción de la Reforma Educativa en América Latina y el Caribe (PREAL), 51.

Darling-Hammond, L. (2012). Educar con calidad y equidad. Los dilemas del siglo XXI. Santiago, Fundación Chile.

Denzin, N., y Lincoln, Y. (2013). Las Estrategias de Investigación Cualitativa. España: Gedisa.

DocenteMás (2019). Manual del Portafolio 2019. Santiago: MINEDUC.

Domingo, A. (2008). Tesis Doctoral: La Práctica Reflexiva en la formación inicial de maestros/as. Evaluación de un modelo. Barcelona.

Domingo, A., Gómez Serés, M., y Zabalza, M. (2014). La práctica reflexiva: Bases, modelos e instrumentos. Madrid: Narcea.

Donoso, S. (2005). «Reforma y Política Educacional en Chile, 1990-2004: el Neoliberalismo en Crisis». Estudios Pedagógicos XXXI, 113-135.

Erazo, S. (2009). «Práctica reflexiva como estrategia de desarrollo profesional: presencia y estructura en reuniones docentes». Educador y Educadores, 12(2).

Eyzaguirre, B. y Le Foulon, C. (2016). La Calidad de la Educación Chilena en Cifras. Santiago: CEP.

Eyzaguirre, S. (Agosto de 2019). Reportaje: La importancia de evaluar y evaluarse.

Flick, U. (2015). Diseño de la Investigación Cualitativa. Madrid: Morata.

García-Huidobro, J.E. y C. Cox (1999). La Reforma Educacional Chilena 1990-1998. Visión de Conjunto. La Reforma Educacional Chilena. Madrid: Editorial Popular, 7-46. 
Gatica, F. y Uribarren, T. (2013). «¿Cómo elaborar una rúbrica?». Investigación en Educación Médica, 61-65.

Gibbs, G. (2012). El análisis de Datos Cualitativos en Investigación Cualitativa. Madrid: Morata.

Gordon, R., Kane, T. \& Steiger, D. (2006). Identifying effective teachers using performance on the job. Washington, D.C.: The Brookings Institution.

Guzmán, I., Marín, R., Zesati, G. y Breach, R. (2012). «Desarrollar y evaluar competencias docentes: estrategias para una práctica reflexiva». Voces y Silencios: Revista Latinoamericana de Educación, 3(1), 22-40.

Korthagen, F. (2010). «La práctica, la teoría y la persona en la formación del Profesorado». Revista Interuniversitaria del Profesorado, 68, 83-101.

Kvale, S. (2011). Las entrevistas en Investigación Cualitativa. Madrid: Morata.

León, M., Manzi, J. y Paredes, R. (2008). «Calidad docente y rendimiento escolar en Chile: Evaluando la evaluación». Encuentro Anual de la Sociedad de Economía de Chile. Viña del Mar.

Martin-Kniep, G. (2001). Portafolios del desempeño de maestros, profesores y directivos: La sabiduría de la práctica. Quilmes: Paidós.

Martínez, C. (1996). Evaluación de Programas Educativos. Madrid: Universidad Nacional de Educación a Distancia.

Ministerio de Educación (23 de Marzo de 2013). DocenteMás. Recuperado de https://www.docentemas.cl/docs/2012/Resultados_Evaluacion_Docente_2012.pdf

Ministerio de Educación (2017). Informe Nacional. Revisión de las politicas educativas en Chile desde el 2004 a 2016. Santiago: MINEDUC.

Ministerio de Educación (2019). Recuperado de http: / / portaldocente.mineduc.cl/portal_docente-web/mvc/inicio/inicio\#!

Murillo, J. (2007). Evaluación del Desempeño y Carrera Profesional Docente. Santiago: Oficina Regional de Educación de la UNESCO. 
Nocetti, A. y Medina, J. (2019). «Significado de reflexión sobre la acción en el estudiantado y sus formadores en una universidad chilena». Revista Educación, 43(1).

Nocetti, A. y Medina, J. (2018). «Condiciones que desencadenan la reflexión docente en el futuro profesor durante sus prácticas de formación». Revista espacios, 39(15), 2.

Nye, B., Konstantopoulos, S. y Hedges, L. (2004). «How large are teacher effects?». Educational Evaluation and Policy Analysis, 26(3), 237-257.

OCDE (2014). Education at a Glance 2014: OCDE Indicators, OCDE Publishing, Paris.

Pérez-Cotapos, M. y Taut, S. (2016). «Adaptación y Pilotaje de un Portafolio para Evaluar Prácticas de Evaluación de Aprendizajes en el Aula en Profesores de Matemática de Segundo Ciclo Básico». Psykhe, 25(1), 1-16.

Perrenoud, P. (2004). Diez nuevas competencias para enseñar. Barcelona: Graó.

Preiss, D., Calgani, E., Espinoza, A., Gómez, D., Grau, V., Guzmán, V. y Volante, P. (2014). «Buenas Prácticas Pedagógicas Observadas en el Aula de Segundo Ciclo Básico en Chile». PSYKHE 23, 1-12.

Rodríguez, G., Gil, J. y García, E. (1996). Metodología de la investigación cualitativa. Málaga: Algibe.

Rosales, C. (1999). Criterios para una Evaluación Formativa: objetivos, contenidos, profesores, aprendizajes, recursos. Madrid: Narcea.

Rubilar, L. (2004). «La Escuela Nacional Unificada». Revista Extramuros, 59-65.

Santelices, M., Galleguillos, P., González, J. y Taut, S. (2015). «Un Estudio sobre la Calidad Docente en Chile: el Rol del Contexto en donde Enseña el Profesor y Medidas de Valor Agregado». Psykhe, 1-14.

Seldin, P. y Annis, L. (1990). «The teaching portfolio». Journal of Staff, Program and Organizational Development, 8, 197- 201. 
Schmelkes, S. (2014). Temas críticos para formular nuevas politicas docentes en América Latina y el Caribe: el debate actual. Santiago: Centro de Estudios de Políticas y Prácticas en Educación (CEPPE), UNESCO.

Schön, D. (1992). La formación de profesionales reflexivos. Barcelona: Paidós.

Sun, Y., Correa, M., Zapata, A. y Carrasco, D. (2011). «Resultados: qué dice la Evaluación Docente acerca de la enseñanza en Chile». Centro de Medición MIDE UC, 91-136.

Tardif, M. (2004). Los saberes del docente y su desarrollo profesional. Madrid: Narcea.

Toledo, G. (2010). Calidad docente y logro escolar: Enfrentando el problema de ordenamiento no aleatorio entre características de profesores y alumnos. Tesis para optar al grado de Magíster en Economía. Santiago: Universidad de Chile.

UNESCO. (2007). Documento de discusión sobre politicas educativas en el marco de la II Reunión Intergubernamental del Proyecto Regional de Educación para América Latina y el Caribe. Santiago: UNESCO.

Vasilachis, I. (2006). Estrategias de Investigación Cualitativa. Gedisa: Barcelona.

Van Manen, M. (1977). «Linking ways of knowing with ways of being practical». Curriculum Inquiry, 6(3), 205-208.

Valdés, H. (2000). «La evaluación del desempeño del docente: un pilar del sistema de evaluación de la calidad de la educación en Cuba». REVISTA OEI. 